\title{
Bearing Damage Detection of BLDC Motors Based on Current Envelope Analysis
}

\author{
Chun-yao Lee and Yu-Hua Hsieh \\ Department of Electrical Engineering, Chung Yuan Christian University, Taoyuan County, 32023, Taiwan \\ cylee@ee.cycu.edu.tw
}

This paper proposes current envelope analysis (CEA) to analyze bearing fault signals in brushless direct current (BLDC) motors, and back propagation neural networks (BPNN) to automatically identify bearing faults. We made sample motors which contained different types of fault, recorded the current signals, and extracted the current features using CEA and Hilbert Huang transform (HHT) for BPNN fault identification. The results indicate that this approach can efficiently identify bearing faults in BLDC motors.

Keywords: Current envelope analysis, back propagation neural networks, Hilbert Huang transform

\section{INTRODUCTION}

A DC MOTOR APPLIED extensively and versatilely is reliable and indispensable industrial equipment. Nevertheless, it can fail, the failure caused by, e.g., improper operation, manufacturing defects and mechanical wear. Different internal motor faults (short circuit of motor leads, interterm short circuits, ground faults, worn out/broken bearings [1-4], broken rotor bars) along with external motor faults are common types of damage that shorten the life span of motors. Many literatures have discussed motor operation. For a quality motor, proper maintenances and applications are important.

A signal analysis technique is important to recognize fault signals [5]-[6]. For example, fast Fourier transform (FFT) [7-8] and wavelet transform (WT) are usually employed for signal analysis [9-12], but their restrictions of selecting a particular mother wavelet will lead to incorrect analysis of signals. Thus, the Hilbert-Huang transform (HHT) proposed by Norden E. Huang, which is composed of empirical mode decomposition (EMD) and Hilbert transform (HT) [13-16], refines traditional methods. HHT can not only be applied to analyze nonlinear and non-stationary signals, but also to improve the problems of traditional analysis methods. As the advantage of the HHT method, the noise problem which may cause inaccurate analysis is discussed in this paper.

Recently, neural network has been extensively applied in, e.g., damage detection, control, and signal processing. The artificial neural network (ANN) has been developed into numerous neural networks for various applications, such as feed-forward network [17-19], probabilistic neural network (PNN) [20-21], and back propagation neural network (BPNN) [22]. All of these neural networks have unique structure, performance, and training methods. Therefore, selecting an appropriate ANN is crucial. BPNN is a supervised neural network which trains with signals of a motor in advance to obtain its weight for the following classification. BPNN only takes extra training time to obtain the weight for promptly completing signal classification of a motor, and BPNN has better accuracy than unsupervised neural networks.
Therefore, this paper measures bearing fault current signals in BLDC motors, extracts features of these signals, and identifies the fault types using BPNN. This approach can prevent unexpected faults, and accelerate fault identification.

\section{CURRENT ENVELOPES AND FEATURE EXTRACTION}

\section{A. Current Envelopes}

Early studies measured motor vibration signals typically with accelerometers, but noises from the environment significantly distort the vibration signals. Our study measures current signals, and effectively identifies earlystage bearing faults using CEA.

CEA is an easy and fast method to identify motor bearing faults. This method determines signal's local maximum values and local minimum values. This approach connects all local maximum values to form upper envelopes, and all local minimum values to form lower envelopes. The flowchart of the study is shown in Fig.1. Fig.2 illustrates upper and lower envelopes of a signal.

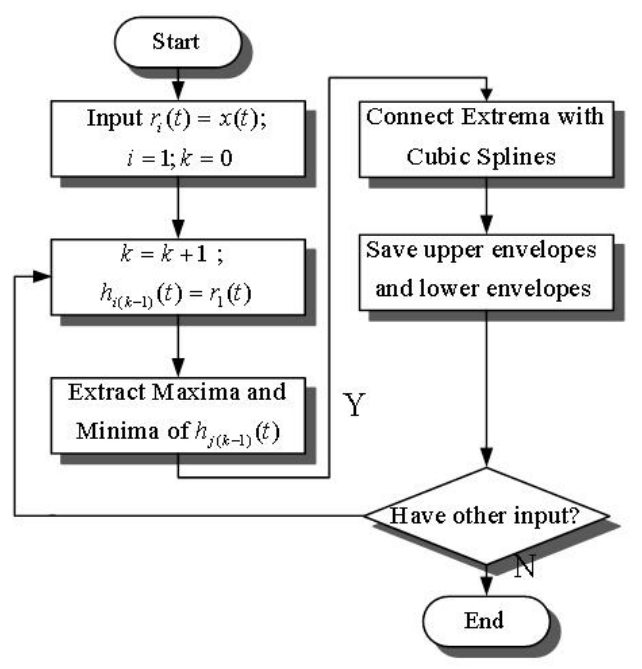

Fig.1. The flowchart of the proposed CEA. 


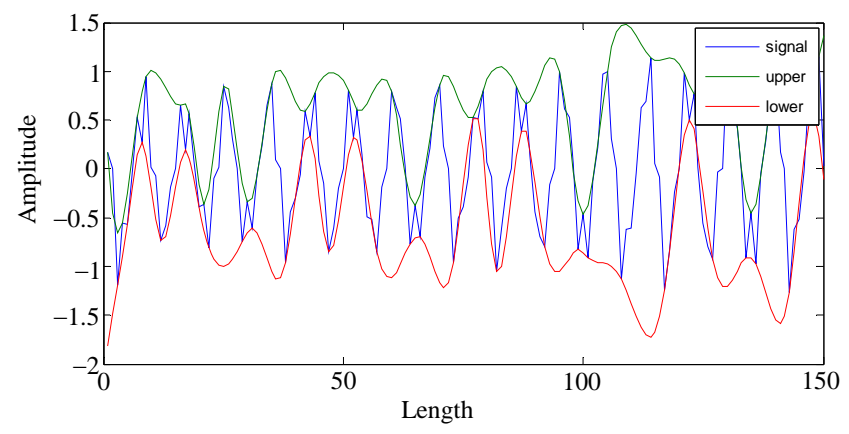

Fig.2. The upper and lower envelopes of a signal.

\section{B. Envelope Feature Extraction}

Neural networks require time to complete their data training. The fewer amount of data means the fewer time the training takes. Hence, feature extraction can significantly reduce the training time and obtain precise results. We analyzed fault signal upper and lower envelopes, calculated their maximum values, minimum values, mean values, root mean square, and 10 energy feature values F1-F10, and applied the 10 feature values to BPNN. The process of the calculation is shown in Fig.3.



Fig.3. The envelope feature extraction.

\section{Hilber huang TRANSFORMER}

The signal analysis, Hilbert Huang transform, was proposed by Dr. Huang E., and is composed of the Hilbert transform and Empirical Mode Decomposition. The method is helpful to the non-stationary and nonlinear signals. First, Empirical Mode Decomposition disintegrates the signal to obtain the intrinsic mode functions and the central tendency that eliminating the noise is effective. Second, intrinsic mode functions obtain instantaneous frequency and instantaneous amplitude of the signal through Hilbert transform, and then get the signal corresponding time, frequency and energy.

\section{Empirical Mode Decomposition (EMD)}

EMD method has been developed from the simple assumption that any signal consists of different simple intrinsic modes of oscillations. Each linear or non-linear mode will be applicable. IMFs must satisfy the following definition:

1. In the whole data set, the number of zero-crossings must either be equal to or differ at most by one.
2. At any point, the mean value of the envelope defined by the local maxima and the envelope defined by the local minima is zero.

An IMF represents a simple oscillatory mode compared with the simple harmonic function. The method is based on removing noise to make the DC motor fault detection easier. The sifting process is as follows:

Step1: Identify all the local maxima and minima of $x(t)$.

Step2: Connect all the maxima by a cubic spline line as the upper envelope.

Step3: The input signal $x(t)$ subtracts the mean envelope $m_{i k}$ to obtain a new signal $h_{i k}$.

Step4: Determine whether $h_{i k}$ isthe IMF, for example with the establishment of its deposit in the $c_{i}(t)$, otherwise repeat step one to step four.

Step5: Separate $c_{i}$ from $x(t)$, we get $r_{i}(t)=x(t)-c_{i}(t) . r_{i}$ is treated as the original data.

Step6: The decomposition process can be stopped when $r_{i}$ becomes a monotonic function.

The flowchart of EMD decomposition is shown in Fig.4. The decomposition process is stopped when $r_{n}(t)$ becomes a monotonic function from which no more IMFs can be extracted. Combine the IMFs and the central tendency of signal as shown in (1).

$$
x(t)=\sum_{i=1}^{n} c_{i}(t)+r_{n}(t),
$$

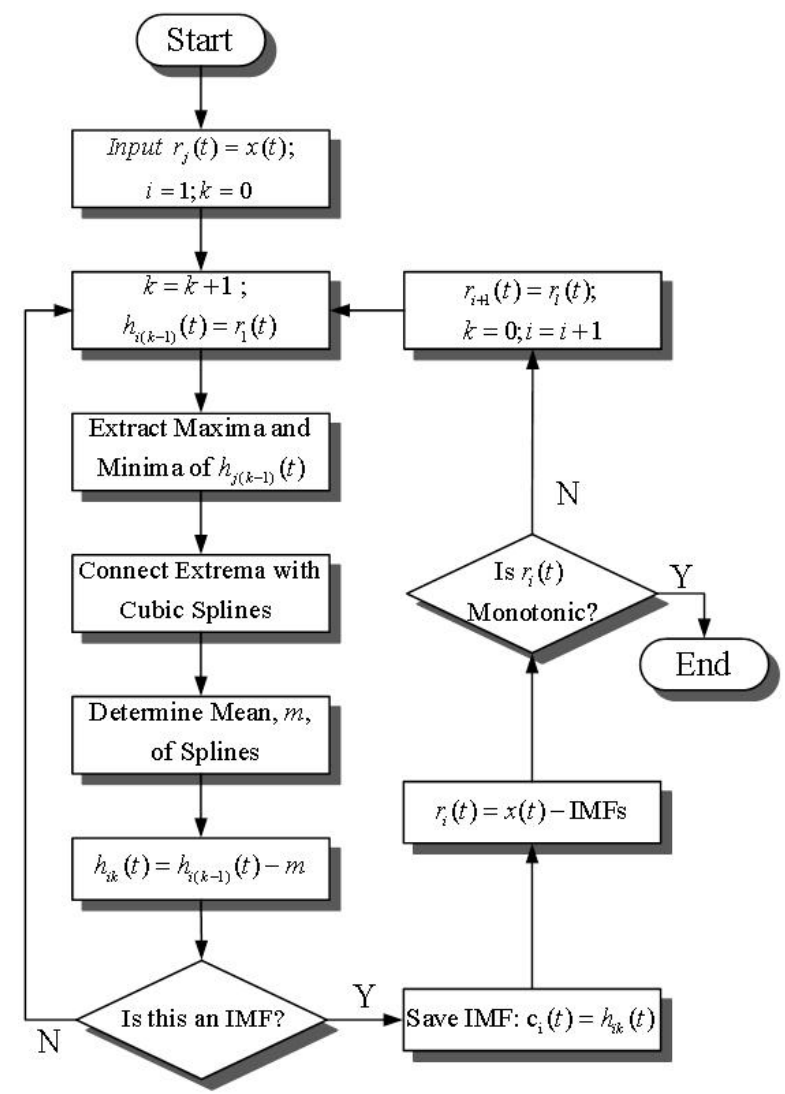

Fig.4. The flowchart of EMD decomposition. 
This paper analyzes BLDC motor fault signals using EMD. Fig.5 demonstrates the IMFs and the tendency function. The $c_{1}$ to $c_{9}$ show IMFs from high frequency to low frequency, respectively, and the $\mathrm{c}_{10}$ shows the tendency function.

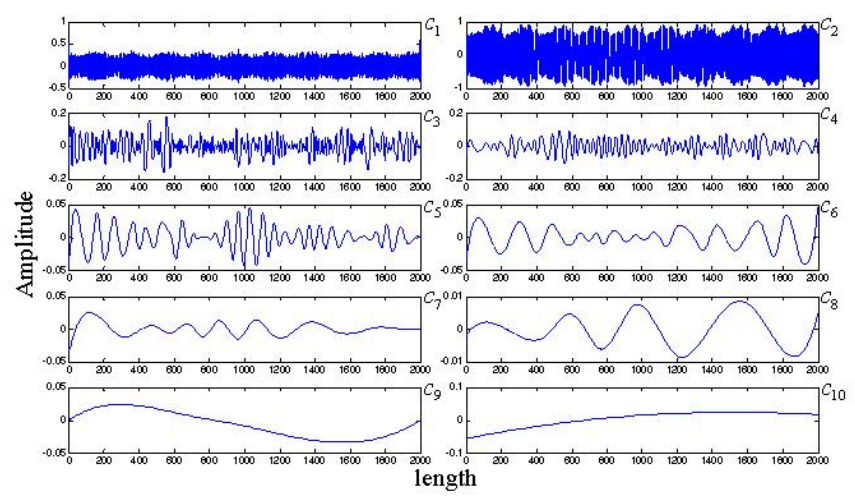

Fig.5. The IMFs of BLDC motor fault signal.

\section{A. Hilbert transform}

Hilbert transform, solving the previous nonlinear and nonstationary signal analysis problems, is a good way to identify motor failure. The conventional Hilbert transform of a continuous signal $c_{i}(t)$ is computed as shown in (2)

$$
H\left[c_{i}(t)\right]=\frac{1}{\pi} \int_{-\infty}^{\infty} \frac{c_{i}\left(t^{\prime}\right)}{t-t^{\prime}} d t^{\prime}
$$

Coupling the complex conjugate pair $H\left[c_{i}(t)\right]$ and $c_{i}(t)$, we get the analytic signal $z(t)$ as shown in (3).

$$
Z_{i}(t)=c_{i}(t)+j H\left[c_{i}(t)\right]=a_{i}(t) e^{j \phi(t)},
$$

Where $a_{\mathrm{i}}(t)$ and $\phi_{i}(t)$ are the instantaneous amplitude and instantaneous phase as shown in (4) and (5)

$$
\begin{gathered}
a_{i}(t)=\sqrt{c_{i}^{2}(t)+H^{2}\left[c_{i}(t)\right]}, \\
\phi_{i}(t)=\arctan \frac{H\left[c_{i}(t)\right]}{c_{i}(t)},
\end{gathered}
$$

The instantaneous frequency is defined as the time derivative of the instantaneous phase as shown in (6)

$$
\omega_{i}(t)=\frac{d \phi_{i}(t)}{d t}
$$

After analyzing IMFs with HT, we attained signal's instantaneous frequency and instantaneous amplitude, and depicted HHT spectrum accordingly. In Fig.6, the Y-axis stands for signal frequency, and the $\mathrm{X}$-axis stands for signal time. Fig.6 differentiates intensity of signal energy using different colors. Therefore the HHT spectrum provides an effective approach to simultaneous observation of distribution of BLDC motor fault signal energy in time and frequency domains.

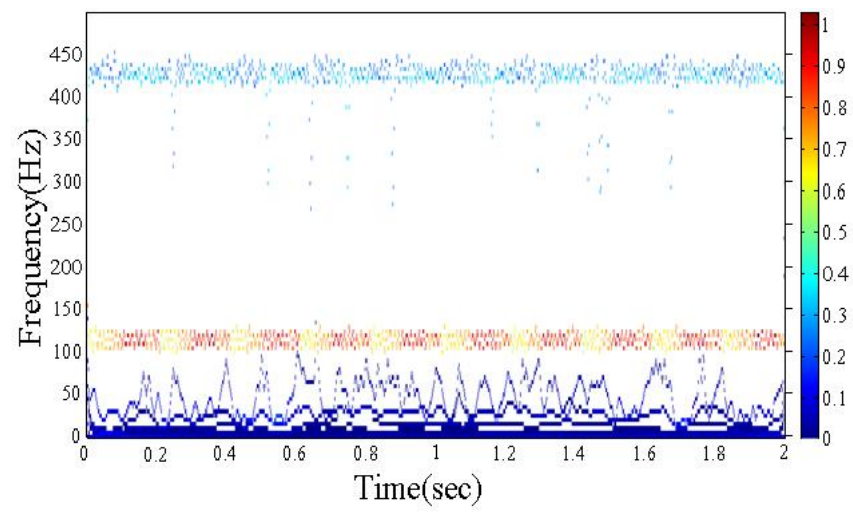

Fig.6. The HHT spectrum.

\section{B. HHT Spectrum Feature Extraction}

Using HHT for fault signals of a motor obtains instantaneous frequency, instantaneous amplitude, and an HT-matrix formed by time, frequency and amplitude to illustrate the HHT spectrum. First, according to the time row, its characteristics including maximum, energy, mean, standard deviation, and root mean square (RMS) can depict five time characteristic curves, namely (1) Tmax, (2) Te, (3) Tmean, (4) Tstd, and (5) Trms. Second, from the frequency row, we find the same five characteristic and obtain curves (6) Fmax, (7) Fe, (8) Fmean, (9) Fstd, and (10) Frms. Finally, we extract the same five characteristics from those ten curves to gain 50 features, from F1 to F50, and each of which will be trained by BPNN. The HHT feature selection is shown in Fig.7.

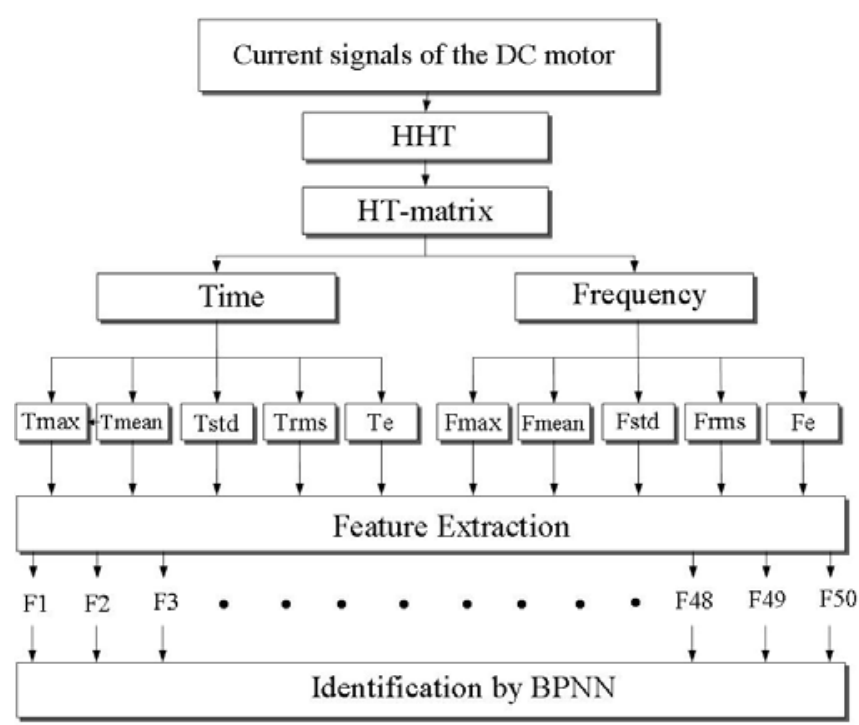

Fig.7. HHT spectrum feature extraction 


\section{BACK PROPAGATION NEURAL NETWORK}

BPNN is a feed-forward network structure [21]-[22], as shown in Fig.8, including input layer and hidden layer. Each neuron only connects to its neighbor layer. In the $k$-th time network training, the error of the $i$-th neuron on output layer is as shown in (7), $t_{i}(k)$ is the expected output, and $x_{i}(k)$ is the real value on $m$-th layer. Shown in (8) is the feedforward phase, in which $s_{m}$ represents the number of neurons on m-th layer, $x_{j}^{m}$ is the $\mathrm{j}$-th neuron on $m$-th layer, $f^{m}(\cdot)$ is activation function, $w_{i, j}^{m}$ is weight, and $b_{j}^{m}$ is bias. The weights of BPNN are updated as shown in (9). The purpose of training BPNN is to minimize the error $F(k)$, as shown in (10).

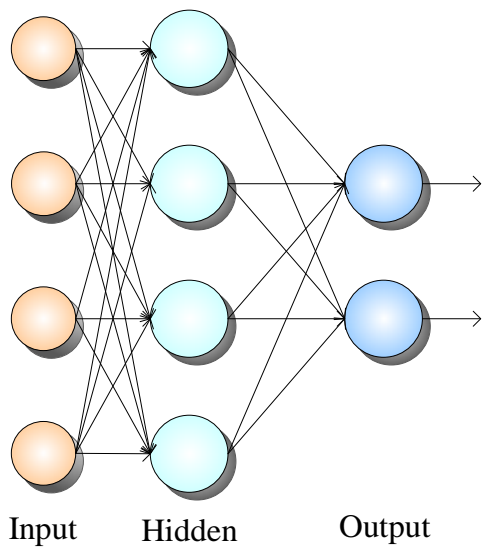

Fig.8. The structure of BPNN.

$$
\begin{gathered}
e_{i}(k)=t_{i}(k)-y_{i}(k), \\
\left\{\begin{array}{l}
x_{i}^{m}=f^{m}\left(\left(\sum_{j=1}^{s^{m-1}} w_{i, j}^{m} x_{i, j}^{m-1}\right)+b_{i}^{m}\right), m \notin \text { input }, \\
x_{i}^{m}=\text { input }, m \in \text { input }
\end{array}\right. \\
\left\{\begin{array}{l}
w_{i, j}^{m}=(k+1)=w_{i, j}^{m}(k)-\alpha e_{i}(k) x_{j}^{m-1}, \\
b_{i, j}^{m}(k+1)=b_{i, j}^{m}(k)-\alpha e_{i}(k) \\
F(k)=\sum_{i \in \text { input }} e_{i}^{2}(k) .
\end{array}\right.
\end{gathered}
$$

\section{MEASUREMENT AND RESULtS}

Our study subject was BL90S motor (24VDC, 150W, 3000rpm) bearing standard (bearing type specification 6001, $12 \mathrm{~mm}$ ID, 28mm OD, $8 \mathrm{~mm}$ depth.) The typical bearing structure includes two homocentric races: 1) Inner race, and 2) Outer race. Rolling elements are tiny balls rolling between two races, and the section of a rolling element is shown in Fig.9(A). To analyze bearing faults, we first made six bearing damage types using the Electrical Discharge Machining (EDM) technique. The six damage bearing types are shown in Fig.9(B): (a) $1.0 \mathrm{~mm}$ holes on both inner race and outer race, (b) a $1.0 \mathrm{~mm}$ hole on inner race, (c) a $1.0 \mathrm{~mm}$ hole on outer race, (d) $0.5 \mathrm{~mm}$ holes on both inner race and outer race, (e) a $0.5 \mathrm{~mm}$ hole on outer race, and (f) a $0.5 \mathrm{~mm}$ hole on inner race. Then we installed these damaged bearings on motors. Fig.10 shows the location of these damaged bearings.

After installation, we measured the fault signals, recorded these current signals with a NI PXI-5422 data recorder. We then analyzed the signal features using HHT and CEA. To obtain the unit of consistency and comparability, we standardized features and applied these features to BPNN to identify motors faults. The system is shown in Fig.11.

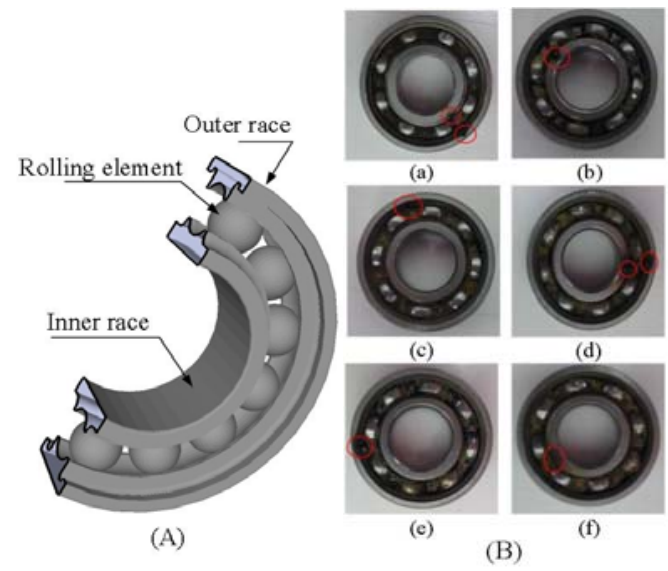

Fig.9. (A) the section of a rolling element (B)The damage bearing in which (a) $1.0 \mathrm{~mm}$ holes on both inner race and outer race (b) $1.0 \mathrm{~mm}$ hole on inner race (c) $1.0 \mathrm{~mm}$ hole on outer race (d) $0.5 \mathrm{~mm}$ holes on both inner race and outer race (e) $0.5 \mathrm{~mm}$ hole on outer race (f) $0.5 \mathrm{~mm}$ hole on inner race.

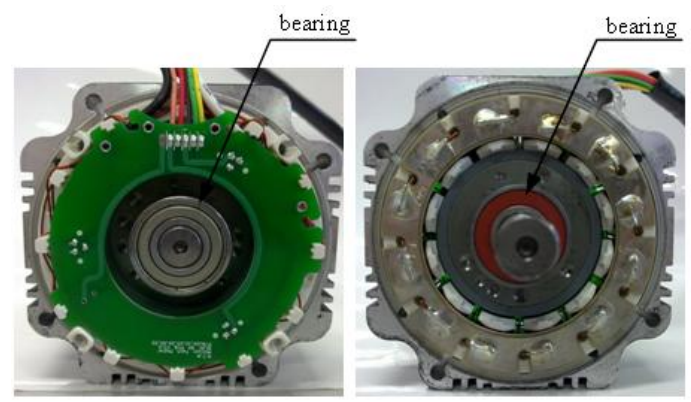

Fig.10. The location of these damaged bearings.

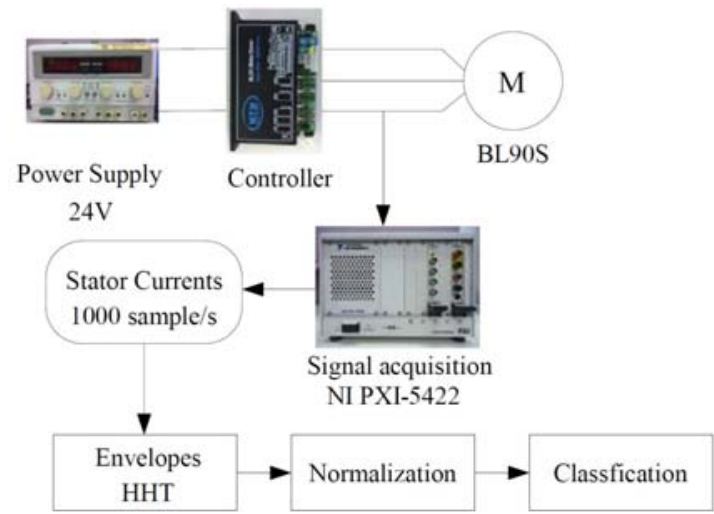

Fig.11. The architecture of the measurement system. 
This study recorded current signals from an intact bearing and six damaged bearings with a NI PXI-5422 recorder. We recorded 50 current signals for each type of bearing and 2,000 points for each current signal. Then we extracted features from each signal using HHT and CEA, and the results are shown in Fig.12 and 13, in which the $\mathrm{X}$-axis represents signal count, the $\mathrm{Y}$-axis represents signal features, and the color represents energy intensity. This indicates that HHT and CEA can successfully analyze signals from intact bearing and six damaged bearing types.

After cross-validating the results, we found that HHT bearing fault identification method can successfully identify average $98 \%$ of motor faults, including intact bearing and the six types of damaged bearing. We further put the system under $20 \mathrm{~dB}$ and $30 \mathrm{~dB}$ noisy environment to validate the robustness of the system. The analysis results indicate that even with 20 and $30 \mathrm{~dB}$ noise the average identification rate is at least $95.6 \%$ and $97.9 \%$, respectively. The identification rates, accuracies, and computing time of HHT for each type of damaged bearing are shown in Table 1 . We also found that the CEA bearing fault identification method can successfully identify average 99\% of motor faults. Even under $30 \mathrm{~dB}$ and $20 \mathrm{~dB}$ noisy environment, the average identification rates are $98.6 \%$ and 98.1 , respectively. The identification rates, accuracies, and computing time of CEA for each type of damaged bearing are shown in Table 2 . Comparing HHT with CEA, we found that CEA overmatch HHT in both identification rate and computing time.

\section{CONCLUSION}

This paper applies Current Envelope Analysis (CEA) to analyze bearing fault signals in BLDC motors and successfully identifies damage types in bearings. We analyzed current signals in BLDC motors using CEA and HHT, and applied results to BPNN to identify fault types. According to our analysis results, CEA average identification rate is $99.1 \%$, and HHT average identification rate is 98\%. CEA requires shorter computing time than HHT does, and maintains relatively high identification rate. To prove the system robustness, we added noise into the system. We found that under 30dB noisy environment CEA and HHT identification rates are $98.6 \%$ and $97.9 \%$, and that under $20 \mathrm{~dB}$ noisy environment their identification rates are 98.1\% and 95.6\%. These results prove that the CEA method can accurately identify fault types in BLDC motors.

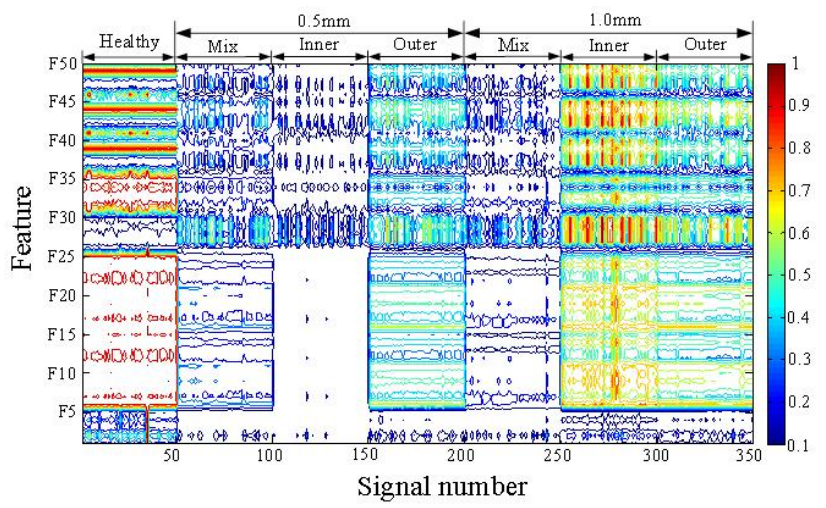

Fig.12. The feature distribution of HHT.

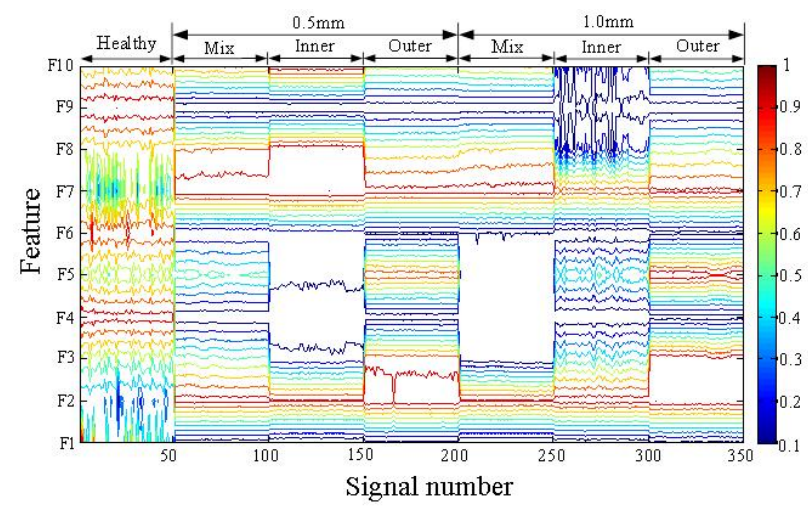

Fig.13. The distribution of CEA feature.

Table 1. The identification rates, accuracies, and computing time of HHT for each type of damaged bearing.

\begin{tabular}{|c|c|c|c|c|c|c|c|c|c|}
\hline \multirow{2}{*}{ Signals Types } & \multirow{2}{*}{ Healthy } & \multicolumn{4}{|c|}{$0.5 \mathrm{~mm}$} & \multicolumn{3}{|c|}{$1.0 \mathrm{~mm}$} & \multirow{2}{*}{$\begin{array}{c}\text { Ave. } \\
\text { Computing time } \\
\text { (sec) }\end{array}$} \\
\cline { 3 - 8 } & & Mix & Inner & Outer & Mix & Inner & Outer & accuracy & \\
\hline \hline Original signals & $100 \%$ & $99.7 \%$ & $100 \%$ & $99.3 \%$ & $99.8 \%$ & $97.4 \%$ & $93.3 \%$ & $\mathbf{9 8 \%}$ & 433 \\
\hline $30 \mathrm{~dB}$ noise & $100 \%$ & $98.7 \%$ & $100 \%$ & $97.8 \%$ & $98.7 \%$ & $99.4 \%$ & $93 \%$ & $\mathbf{9 7 . 9 \%}$ & 440 \\
\hline $20 \mathrm{~dB}$ noise & $100 \%$ & $95.3 \%$ & $100 \%$ & $99.4 \%$ & $94.6 \%$ & $94.4 \%$ & $94.1 \%$ & $\mathbf{9 5 . 6 \%}$ & 436 \\
\hline
\end{tabular}

Table 2. The identification rates, accuracies, and computing time of CEA for each type of damaged bearing.

\begin{tabular}{|c|c|c|c|c|c|c|c|c|c|}
\hline \multirow{2}{*}{ Signals } & \multirow{2}{*}{ Types } & \multicolumn{3}{|c|}{$0.5 \mathrm{~mm}$} & \multicolumn{3}{|c|}{$1.0 \mathrm{~mm}$} & \multirow{2}{*}{$\begin{array}{c}\text { Ave. } \\
\text { Computthy }\end{array}$} \\
\cline { 3 - 9 } & & Mix & Inner & Outer & Mix & Inner & Outer & $\begin{array}{c}\text { accuracy } \\
\text { (sec) }\end{array}$ \\
\hline \hline Original signals & $100 \%$ & $100 \%$ & $100 \%$ & $100 \%$ & $100 \%$ & $100 \%$ & $93.6 \%$ & $\mathbf{9 9 \%}$ & 89 \\
\hline $30 \mathrm{~dB}$ noise & $100 \%$ & $99 \%$ & $100 \%$ & $100 \%$ & $100 \%$ & $100 \%$ & $91.7 \%$ & $\mathbf{9 8 . 6 \%}$ & 93 \\
\hline $20 \mathrm{~dB}$ noise & $100 \%$ & $98.6 \%$ & $100 \%$ & $98.9 \%$ & $100 \%$ & $100 \%$ & 97.2 & $\mathbf{9 7 . 2 \%}$ & 94 \\
\hline
\end{tabular}




\section{ACKNOWLEDGMENT}

This research was supported by the National Science Council of the Republic of China under Grant No. 99-2632E-033-001-MY3.

\section{REFERENCES}

[1] Altug, S., Chow, M.Y., Trussell, H.J. (1999). Fuzzy inference systems implemented on neural architectures for motor fault detection and diagnosis. IEEE Transactions on Industrial Electronics, 46 (6), 10691079.

[2] Manoharan, S.C., Veezhinathan, M., Ramakrishnan, S. (2008). Comparison of two ANN methods for classification of spirometer data. Measurement Science Review, 8 (3), 53-57.

[3] Hasan, M.A., Reaz, M.B.I. (2012). Hardware prototyping of neural network based fetal electrocardiogram extraction. Measurement Science Review, 12 (2), 52-55.

[4] Naik, G.R., Kumar, D.K., Arjunan, S.P. (2010). Pattern classification of Myo-Electrical signal during different Maximum Voluntary Contractions: A study using BSS techniques. Measurement Science Review, 10 (1), 1-6.

[5] Hussain, M.S., Mamun, M. (2012). Effectiveness of the wavelet transform on the surface EMG to understand the muscle fatigue during. Measurement Science Review, 12 (1), 28-33.

[6] Phinyomark, A., Limsakul, C., Phukpattaranont, P. (2011). Application of wavelet analysis in EMG feature extraction for pattern classification. Measurement Science Review, 11 (2), 45-52.

[7] Kijewski-Correa, T., Kareem, A. (2006). Efficacy of Hilbert and wavelet transforms for time-frequency analysis. Journal of Engineering Mechanics, 132 (10), 1037-1049.

[8] Phinyomark, A., Limsakul, C., Phukpattaranont, P. (2011). On Hilbert-Huang transform approach for structural health monitoring. Journal of Intelligent Material Systems and Structures, 17 (8), 721-728.

[9] Phinyomark, A., Limsakul, C., Phukpattaranont, P. (2006). A new envelope algorithm of Hilbert-Huang transform. Mechanical Systems and Signal Processing, 20 (8), 1941-1952.

[10] daSilva, A.M., Povinelli, R., Demerdash, N.A.O. (2008). Induction machine broken bar and stator shortcircuit fault diagnostics based on three-phase stator. IEEE Transactions on Industrial Electronics, 55 (3), 1310-1318.
[11] Rivas, E., Burgos, J.C., Garcia-Prada, J.C. (2009). Condition assessment of power OLTC by vibration analysis using wavelet transform. IEEE Transactions on Power Delivery, 24 (2), 687-694.

[12] Chow, M.-Y., Sharpe, R.N., Hung, J.C. (1983). On the application and design of artificial neural networks for motor fault. IEEE Transactions on Industrial Electronics, 40 (2), 189-196.

[13] Tamura, S., Tateishi, M. (1997). Capabilities of a fourlayered feedforward neural network: Four layers versus three. IEEE Transactions on Neural Networks, 8 (2), 189-196.

[14] Huang, G.-B., Chen, L., Siew, C.-K. (2012). Universal approximation of extreme learning machine with adaptive growth of hidden nodes. IEEE Transactions on Neural Networks Learning System, 23 (2), 365371.

[15] Tripathy, M., Maheshwari, R.P., Verma, H.K. (2012). Power transformer differential protection based on optimal probabilistic neural network. IEEE Transactions on Power Delivery, 25 (1), 102-112.

[16] Perera, N., Rajapakse, A. (2012). Recognition of fault transients using a probabilistic neural-network classifier. In IEEE Power and Energy Society General Meeting, 24-29 July 2011.

[17] Wei, W., Feng, G., Li, Z., Xu, Y. (2005). Deterministic convergence of an online gradient method for BP neural networks. IEEE Transactions on Neural Networks, 16 (3), 533-540.

[18] Cheng, J.S., Yu, D.J., Tang, J.S. (2008). Deterministic convergence of an online gradient method for BP neural networks. Mechanism and Machine Theory, 43 (6), 712-723.

[19] Yang, R.Q., Gao, X. (2006). Hilbert-Huang transformbased vibration signal analysis for machine health monitoring. IEEE Transactions on Instrumentation and Measurement, 55 (6), 2320-2329.

[20] Shukla, S., Mishra, S., Singh, B. (2009). Empiricalmode decomposition with Hilbert transform for power-quality assessment. IEEE Transactions on Power Delivery, 21 (4), 2159-2165.

[21] Burgsteiner, H. (2006). Training networks of biological realistic spiking neurons for real-time robot control. IEEE Transactions on Power Delivery, 19 (7), 741-752.

[22] Selaimia, Y., Moussaoui, A., Abbassi, H.A. (2006) Multi neural networks based approach for fault detection and diagnosis of a DC-Motor. Neural Network World, 16, 369-379.

Received July 19, 2012. Accepted November 20, 2012. 